\title{
FOCOS E FATORES ASSOCIADOS AO APARECIMENTO DE LEISHMANIOSE TEGUMENTAR AMERICANA (LTA) E LEISHMANIOSE VISCERAL (LV) NO CARIRI CEARENSE
}

\section{FOCUS AND FACTORS ASSOCIATED WITH THE ONSET OF AMERICAN CUTANEOUS LEISHMANIASIS AND VISCERAL LEISHMANIASIS IN CARIRI FROM CEARÁ}

\author{
*OLIVEIRA, Alexcian R. de. \\ Secretaria de Educação do Estado do Ceará \\ FERNANDES, Cláudia A. \\ Laboratório de Análises Clínicas do Crato
}

Recebido em: 21/03/2014; aceito: 06/11/2014; publicado em 19/11/2014

\section{RESUMO}

Em decorrência de surtos epidêmicos no Ceará, em especial no Cariri Cearense, faz-se relevante o mapeamento de casos por localidade, sendo este o objetivo principal deste trabalho. Os dados foram acessados pelo "Sinan Net", analisados pelos programas Microsoft Office Execel 2007, epiinfo (versão 3.5.2) e stata (versão 10) e relacionados a outras bibliografias. Os resultados foram que o desmatamento, migração e urbanização são características identificadas nas áreas afetadas, sendo possível identificar 3 municípios como mais acometidos com mais de 70\% dos casos de LTA e LV e outros 3 como um possível novo "cluster" de transmissão. Conclui-se que na região a Leishmaniose é autóctone e a análise do quantitativo de casos demonstra que houve uma maior disseminação da patologia em determinadas localidades suscitando necessidade de melhor acompanhamento das áreas mais afetadas.

Palavras-chave: leishmania; transmissão; aglomeração; endêmico; negligenciada

\begin{abstract}
Because of epidemic outbreaks in Ceará, Cariri from Ceará in particular, is relevant the mapping of cases by location to be this is the main objective of this work. Data were accessed by the "Sinan Net", analyzed with Microsoft Office Execel2007, epiinfo (version 3.5.2) and Stata (version 10) and bibliographies related. The results was deforestation, urbanization and migration characteristics are identified in the affected areas, it did possible to identify three towns most affected above $70 \%$ of cases of "LTA" and "LV" and other three as a possible new "cluster" of transmission. The conclusion was in region Leishmaniasis is autochthonous and the quantitative analysis of the cases shows that there was a greater spread of the disease in certain localities pointing need for better accompany of most affected areas.
\end{abstract}

Keywords: leishmania; transmission; agglomeration; endemic; neglected

\footnotetext{
${ }^{*}$ ALEXCIAN RODRIGUES DE OLIVEIRA. Endereço para correspondência: Avenida José Alves de Figueiredo, nº1216, apt ${ }^{\circ}$ 103, Bairro Seminário, Crato-Ceará, Brasil. CEP:63113-810. Telefone para contato:(88)9910-4194
} 


\section{INTRODUÇÃ̃O}

A Leishmaniose é uma doença complexa e disseminada pelo mundo que dependendo da resposta imune do infectado pode acometer regiões distintas dos tecidos parasitados e por consequência exibe diferentes formas clínicas causadas por cerca de 20 espécies de protozoários patogênicos ${ }^{1}$. Também pode ser considerada uma doença negligenciada já que está associada a má nutrição, condições precárias de moradia, baixa escolarização e escassez de recursos para sobrevivência, além de demonstrar peculiaridade com áreas de desmatamento, regiões com urbanizações recentes ou acompanhadas de processos migratórios ${ }^{2}$.

A Leishmaniose pode ser causa de letalidade em larga escala caso medidas de controle não sejam tomadas, a exemplo do que aconteceu na Região Africana conhecida como Sudão (atualmente dois países "Sudão" e "Sudão do Sul"), entre 1984 e 1994, período no qual morreram cerca de 100.000 pessoas de uma população total de 300.000 na década citada ${ }^{3}$. Sua distribuição pelo mundo é bastante heterogênea no que se refere a forma Visceral, enquanto que na forma cutânea a distribuição de casos se dá de maneira mais uniforme $e^{4}$ O Brasil também identificou diversos focos da doença no decorrer dos últimos anos ${ }^{5}$.

Nas Américas já foram reconhecidas 11 espécies de Leishmanias cutâneas, sendo que no Brasil foram identificadas 7, das quais 6 eram do subgênero "Viannia". No que diz respeito a Leishmaniose visceral a espécie "Leishmania chagasi”, um parasito intracelular obrigatório sob forma aflagelada ou amastigota das células do sistema fagocítico mononuclear tem sido a espécie comumente envolvida nessa variação da patologia ${ }^{5}$.

Em virtude de sua endemicidade na América Latina, em especial no Brasil, considerando a necessidade de padronizar procedimentos normativos relacionados a notificação compulsória e a vigilância epidemiológica, órgãos oficiais como Ministério da Saúde adotaram a classificação agrupada como "Leishimaniose Tegumentar Americana" (LTA) e "Leishmaniose Visceral" (LV) em toda rede pública e privada para os casos de Leishmaniose no país ${ }^{6}$.
Característica peculiar a transmissão do protozoário é a presença de vetores dípteros tendo como destaque o gênero Lutzomyia sp denominado também como flebotomíneo e conhecido popularmente como mosquito palha, borrachudo, tatuquiras ou birigui dentre outros envolvido no ciclo de transmissão ${ }^{7}$.

Devido o aumento recente de casos de Leishmaniose em algumas regiões do Brasil e mais especificamente no estado do Ceará, faz-se relevante o mapeamento do quantitativo de casos por localidade, assim como a análise das primeiras cartografias epidemiológicas como se pode observar na figura $1^{8}$.

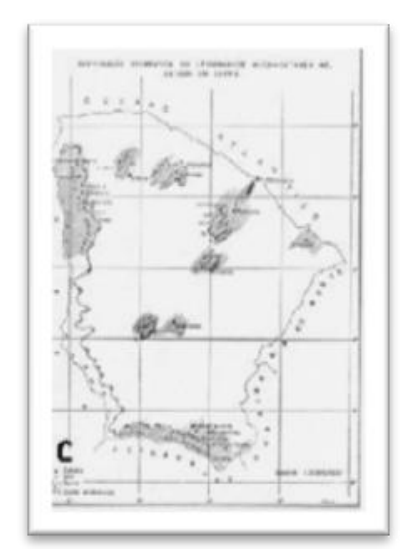

Figura 1- Mapa da distribuição de Leishmaniose cutânea no estado do Ceará, Brasil em 1952.

A distribuição de casos no período sugere associação entre migração das dezenas de milhares de pessoas entre o Ceará e Região Amazônica decorrente das secas e dos surtos epidêmicos como os que ocorreram entre 1800 e $1900^{9}$ que ao retornarem trouxeram consigo essa doença que emergiu como um importante problema de saúde pública.

Desde as últimas seis décadas é possível observar que o Cariri Cearense se tornou uma região endêmica de casos de Leishmaniose, provavelmente por suas características físicas e sociais, pois situa-se na Região extremo sul do estado fazendo fronteira com outros 3 estados (Piauí, Pernambuco e Paraíba) formando uma mesorregião de 28 municípios constituídos, comportando uma das duas regiões metropolitanas do estado, a qual está nos entornos da "Floresta Nacional do Araripe (FLONA)" 
também chamada de "Chapada do Araripe" uma extensa área de 38.262 hectares e perímetro de cerca de 138 quilômetros ${ }^{10}$.

É considerada um oásis, pois seu subsolo apresenta um extenso reservatório de água, com espécies diversas de vegetais e animais vivendo em suas proximidades. A economia é diversifica e bem distribuída com indústrias, agricultura e criação de animais variados; os recursos hídricos tem disposição contínua durante todo o ano (não em abundância), sendo mais bem disponibilizados que as demais cidades circunvizinhas, principalmente nas cidades de Santana do Cariri, Nova Olinda, Farias Brito, Caririaçu, Missão velha, Jardim, Juazeiro do Norte, Crato e Barbalha que concentram em torno de 500mil habitantes.

Dispõe de diversas faculdades públicas e particulares, incluindo cursos de saúde como Medicina, Enfermagem, Farmácia, Fisioterapia, Biomedicina e Biologia que contribuem com estudos epidemiológicos da região a exemplo de relatos ${ }^{11}$ no qual caracterizaram doentes de uma cidade próxima em surto epidêmico; além do mais há um sistema de saúde de vigilância permanente com emergências, atenções básicas implantadas pelas cidades e a presença de 2 das 6 unidades do Laboratório Central do Estado-LACEN ${ }^{12}$ os quais auxiliam na otimização do processo diagnóstico. Como se pode observar nesta região há a formação de um "cluster" de municípios com densidades demográficas que se destacam em relação aos demais, cujo valor do indicador varia em torno de 50 a 1.000 hab. $/ \mathrm{km}^{2}$, a exemplo do município de Juazeiro do Norte $\left(1.005,54\right.$ hab. $\left./ \mathrm{km}^{2}\right)$ que deteve a maior densidade demográfica do estado depois da capital Fortaleza $(7.815,70)$ e Maracanaú $(1.984,45)^{13}$.

Os municípios de Juazeiro do Norte, Crato e Barbalha tem recebido destaque no que diz respeito ao aumento de casos de Leishmaniose nos últimos anos, em especial a "LTA", principalmente os dois últimos municípios por manifestarem surtos.

\section{METODOLOGIA}

Por meio de dados de domínio público baixados de sites da Secretaria de Vigilância Sanitária (SVS) do Ministério da Saúde (MS) e informações complementares em bibliografias disponíveis procurou-se caracterizar os principais locais com alta prevalência de Leishmaniose (Tegumentar America e Visceral) em determinadas localidades e fatores associados, mais especificamente no Cariri Cearense que são assistidas pelas $19^{\mathrm{a}}, 20^{\mathrm{a}}$ e $21^{\mathrm{a}}$ Coordenadorias Regionais de Saúde do estado para se identificar possíveis causas que propiciaram a epidemia de Leishmanias em determinadas áreas nos últimos anos. Os dados foram acessados no dia 07 de janeiro de 2014 pelo site:<http://dtr2004.saude.gov.br/sinanweb/index.php>.

As análises dos dados foram feitas pelos programas Microsoft Office Execel2007, epiinfo (versão 3.5.2) e stata (versão 10).

A partir das informações obtidas procurou-se evidenciar aspectos dos portadores das patologias.

\section{RESULTADOS}

Os casos de Leishmaniose confirmados positivos por municípios foram: notificados na SVS/MS; estão disponíveis a partir do ano de 2007 e correspondem ao ano de notificação, dos quais foram excluídos os casos não residentes no Brasil, assim como as duplicidades de casos; os casos foram validados pela Gerência Técnica de Leishmanioses, pela Coordenação Geral de Vigilância de Doenças Transmitidas por Vetores e Antropozoonoses (CGDT) e pelo Departamento de Vigilância Epidemiológica (DEVEP) ligados a SVS/MS; os dados foram atualizados em 2009, 2010, 2011 e 2013, sendo que os de 2013 foram atualizados em 01/08/2013 e estão sujeitos à revisão.

A análise dos dados evidenciou que algumas cidades que compõe a região tiveram uma incidência maior de casos que outras nos últimos 7 anos tanto para LTA (tabela 1) como para LV (tabela 2) o que salientou a característica heterogênea da propagação da doença. 


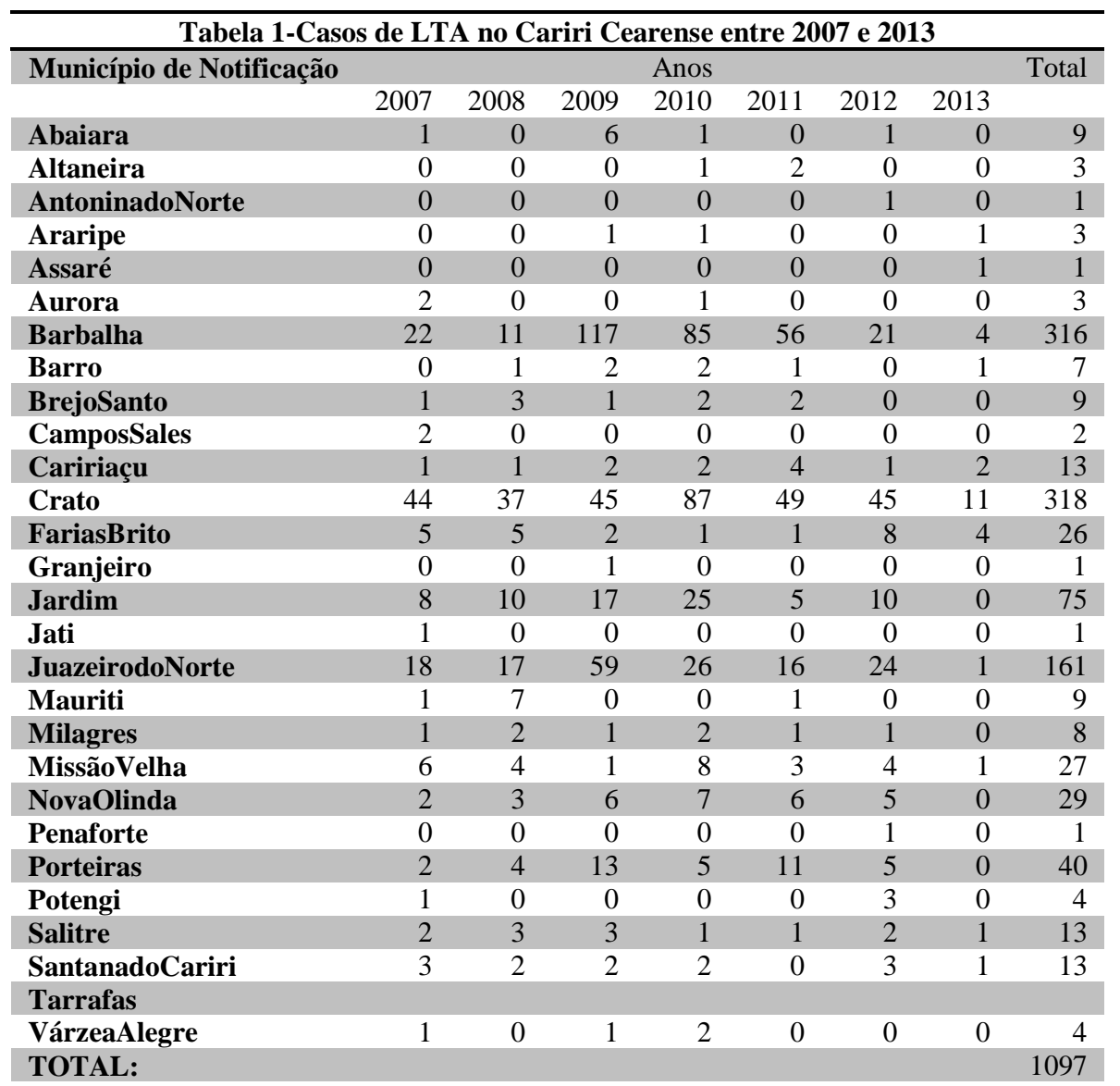

Fonte dos dados: SinanNet/DATASUS

\begin{tabular}{|c|c|c|c|c|c|c|c|c|}
\hline \multicolumn{9}{|c|}{ Tabela 2-Casos de LV no Cariri Cearense entre 2007 e 2013} \\
\hline \multirow{2}{*}{ Município de Notificação } & & & & Anos & & & & Total \\
\hline & 2007 & 2008 & 2009 & 2010 & 2011 & 2012 & 2013 & \\
\hline Abaiara & 0 & 0 & 0 & 0 & 1 & 1 & 0 & 2 \\
\hline Altaneira & 0 & 1 & 0 & 0 & 0 & 0 & 0 & 1 \\
\hline AntoninadoNorte & 0 & 0 & 1 & 0 & 0 & 0 & 0 & 1 \\
\hline Araripe & 0 & 0 & 0 & 1 & 2 & 0 & 0 & 3 \\
\hline Assaré & 1 & 0 & 1 & 1 & 0 & 1 & 1 & 5 \\
\hline Aurora & 0 & 0 & 0 & 0 & 0 & 1 & 0 & 1 \\
\hline Barbalha & 31 & 27 & 25 & 18 & 10 & 11 & 3 & 125 \\
\hline Barro & 0 & 1 & 2 & 1 & 0 & 3 & 0 & 7 \\
\hline BrejoSanto & 4 & 3 & 5 & 4 & 10 & 4 & 2 & 32 \\
\hline CamposSales & 0 & 0 & 1 & 1 & 0 & 0 & 0 & 2 \\
\hline Caririaçu & 0 & 1 & 0 & 0 & 0 & 1 & 0 & 2 \\
\hline Crato & 10 & 13 & 9 & 7 & 13 & 5 & 1 & 58 \\
\hline FariasBrito & 2 & 0 & 1 & 0 & 0 & 1 & 2 & 6 \\
\hline \multicolumn{9}{|l|}{ Granjeiro } \\
\hline \multicolumn{9}{|l|}{ Jardim } \\
\hline Jati & 0 & 0 & 0 & 1 & 0 & 0 & 0 & 1 \\
\hline JuazeirodoNorte & 33 & 22 & 23 & 16 & 8 & 13 & 4 & 119 \\
\hline Mauriti & 0 & 5 & 3 & 5 & 7 & 5 & 0 & 25 \\
\hline Milagres & 0 & 2 & 3 & 1 & 4 & 0 & 2 & 12 \\
\hline MissãoVelha & 0 & 0 & 0 & 0 & 1 & 0 & 0 & 1 \\
\hline NovaOlinda & 1 & 1 & 3 & 2 & 2 & 1 & 2 & 12 \\
\hline Penaforte & 0 & 0 & 1 & 0 & 0 & 0 & 0 & 1 \\
\hline Porteiras & 1 & 0 & 0 & 0 & 1 & 1 & 0 & 3 \\
\hline Potengi & 1 & 0 & 0 & 1 & 0 & 0 & 0 & 2 \\
\hline Salitre & 1 & 0 & 0 & 0 & 1 & 0 & 1 & 3 \\
\hline SantanadoCariri & 0 & 1 & 1 & 0 & 0 & 0 & 0 & 2 \\
\hline Tarrafas & 0 & 0 & 0 & 0 & 0 & 1 & 0 & 1 \\
\hline VárzeaAlegre & 0 & 0 & 0 & 1 & 0 & 0 & 0 & 1 \\
\hline TOTAL: & & & & & & & & 428 \\
\hline
\end{tabular}


Uma análise gráfica da distribuição de casos por municípios demonstra mais nitidamente que Barbalha,

Crato e Juazeiro do Norte foram as cidades mais acometidas pela LTA (figura 2) e que os casos de LV (figura 3) também demonstram afinidade para este ranking dentre todas as 28 cidades circunvizinhas.

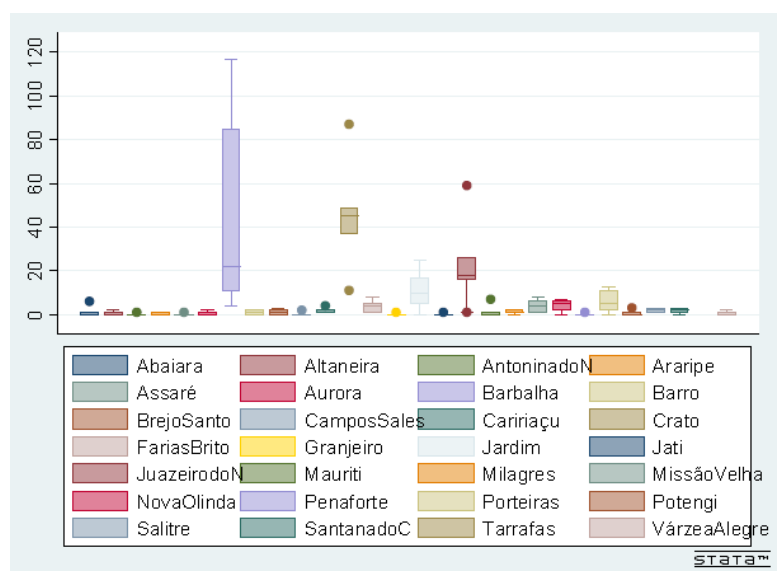

Figura 2- Boxplot de distribuição de casos absolutos de LTA no Cariri Cearense 2007-2013.

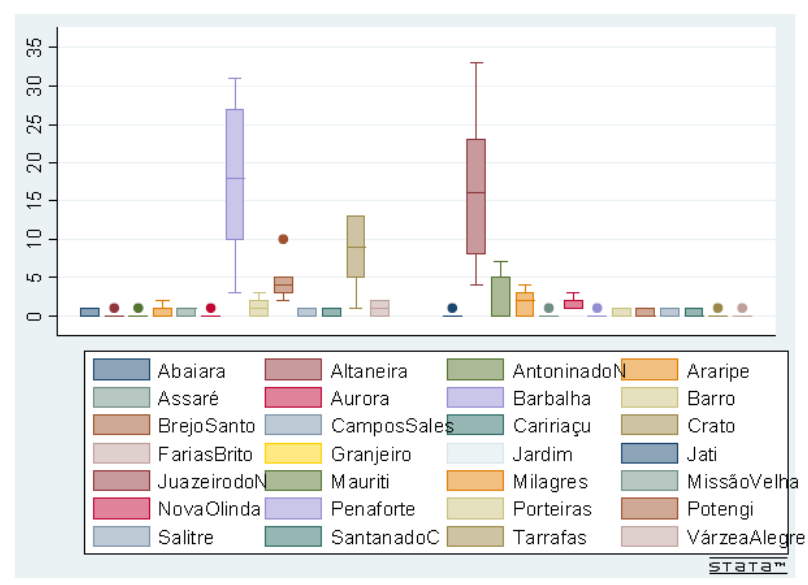

Figura 3- Boxplot de distribuição de casos absolutos de LV no Cariri Cearense 2007-2013.

Do total de casos na série histórica estudada observou-se que Barbalha apresentou 28,80\% dos casos de LTA e 29,20\% dos casos de LV; Crato $29 \%$ de LTA e $13,55 \%$ de LV; e Juazeiro do Norte $14,67 \%$ de LTA e $27,80 \%$ de $\mathrm{LV}$, ou seja, as referidas cidades foram responsáveis por mais de $\mathbf{7 0 \%}$ dos casos de LTA e LV de toda região sul do estado nos 7 anos de notificação compulsória.

Ao se considerar o tamanho da população destas três cidades: Barbalha com 57.818 hab, Crato com 126.591hab e Juazeiro do Norte com 261.289 hab (IBGE, 2014) é possível identificar a população mais agredida pelo parasito por meio da taxa de incidência $\left(\mathrm{n}^{\mathrm{o}}\right.$ acometidos por forma clínica $/ \mathrm{n}^{\circ}$ habitantes no município $\mathrm{x}$ 100.000) (DATASUS, 2014) em cada município no período, evidenciando Barbalha como a mais afetada com uma taxa de 546 casos de LTA e 216 casos de LV por 100mil habitantes, seguida de Crato com taxas de 251 (LTA) e 46 (LV) e Juazeiro do Norte com 61(LTA) e 46(LV) em relação aos 7 anos analisados.

\section{DISCUSSÃO}

É interessante observar que os casos de LTA superam quase que o triplo dos casos de LV, despertando atenção para essa manifestação da patologia, principalmente em Barbalha, Crato e Juazeiro do Norte e que os anos de 2009 e 2010 foram mais propícios para propagação de casos cuja quantidade aumentou significativamente sugerindo o aparecimento de novos fatores de transmissão.

A proximidade geográfica de Crato e Barbalha com a Chapada do Araripe coincide com a propensão da Leishmaniose e o habitat florestal, potencializando a disseminação da doença, já que é de conhecimento notório que existem outros hospedeiros além do homem e o mosquito como reservatórios com importante papel no ciclo de transmissão do protozoário ${ }^{15}$. A proximidade da floresta que também dispõe de vários parques aquáticos atrai uma diversidade enorme de pessoas constantemente (seja por romaria ou lazer) e uma ocupação imobiliária sem precedentes com construção de chácaras e residências para servir de refúgio da vida urbana expondo indivíduos a fatores de risco e transmissão, principalmente aqueles mais vulneráveis como "analfabetos/semianalfabetos" e "agricultores" que tem sido mais acometidos pela patologia nestas localidades ${ }^{11}$.

A invasão de áreas recém desmatadas sugere a ação do homem como fator significativo na expansão da epidemia $^{14}$ e direciona a atenção a grupos residentes nestas localidades por apresentarem risco mais elevado de adquirir o protozoário em função de suas vulnerabilidades de moradia com casas pouco estruturadas e vulnerabilidades comportamentais com criação de animais que sirvam de 
possíveis hospedeiros e/ou negligencia dos primeiros sintomas.

Levando-se em consideração o princípio da possibilidade do aparecimento de novos vetores, a eventualidade de outros flebotomíneos e/ou hospedeiros estarem envolvidos na transmissão deve ser cogitada, contudo a carência de investigações na região a esse respeito limita maiores analogias.

O aparecimento de casos de LTA em Juazeiro do Norte corrobora com a tendência de urbanização da endemia, fato já constatado em outras cidades Brasileiras, assim como países afora em virtude do fluxo não apenas migratório, mas também de sua interação constante dos residentes e a região, ainda mais esta cidade que tem se destacado com o recebimento de grandes empreendimentos públicos e privados e junto com estes uma nova carga genética e social que vem se incorporando e modificando hábitos locais ${ }^{16}$.

Os casos de LV se deram em menor número quando comparados aos de LTA, mesmo assim despertam preocupação quanto a presença desta forma clínica na região, já que em todos os municípios estudados foram notificadas sua presença, com exceção de Granjeiro e Jardim que não apresentaram nenhum caso enquanto toda região no entorno tenha relatado notificações no decorrer dos 7 anos estudados.

Juazeiro do Norte se destaca com uma incidência absoluta de 119 casos de LV superando qualquer outra cidade, inclusive Barbalha e Crato evidenciadas como epidêmicas para a outra forma clínica e subsequentes para a forma manifestada em questão. Popularmente chamada de calazar, a LV tem sido alvo de campanhas educativas e de campanhas por diagnósticos via busca ativa na cidade mais

\section{CONSIDERAÇÕES FINAIS}

Vale salientar que a Leishmaniose nem sempre esteve presente no Ceará, mas nela se fixou e tornou-se um problema de saúde púbica que vem se agravando.

No Cariri Cearense a Leishmaniose tornou-se autóctone e subnotificações repercutem numa maior populosa da região metropolitana ${ }^{17}$, ainda porque é uma doença que tem levado a muitos óbitos no Ceará ${ }^{18}$, outra doença endêmica da região. O aumento da preocupação com a disseminação dessa forma patológica por parte do poder público associado ao aumento crescente de casos evidencia que a epidemia vem ganhando espaço de convivência entre residentes e seu controle se tornou difícil. Esta situação remete a introdução de estratégias de ação em campo, como o que já fora utilizado no passado com outras moléstias ${ }^{19}$, tipo identificação e contenção de focos pela cidade para futura limitação da transmissão e posterior erradicação.

Outra indiscrição que a analise dos dados chama atenção são 3 cidades: Mauriti (45.640habitantes), Brejo Santo (47.218habitantes) e Milagres (28.287habitantes) sendo as únicas a notificar quantitativo de casos em torno do primeiro quartil da distribuição da cidade mais atingida e o que é coincidente, fazem fronteiras entre si, fato que embora não possa determinar a classificação de um novo "cluster" potencializa sua formação caso medidas de contenção da epidemia não sejam tomadas já que o contexto social tendência o aumento do número de casos.

A diversidade de espécies de Leishmanias associada à capacidade de resposta imunitária de cada indivíduo e a infecção está relacionada com as várias formas clínicas $^{20}$. Também é de conhecimento notório que elementos clínicos e epidemiológicos contribuem substancialmente para o diagnóstico da doença ${ }^{21}$, assim como o não diagnóstico que pela confusão dos sintomas com outras doenças por semanas ou meses leva a subnotificações. Essa circunstancia de não diagnóstico poderá ser avaliada quando os dados oficiais forem atualizados futuramente no Sistema Nacional de Agravos de Notificações (SINANNet) em função da variação do número de casos notificados no período.

disseminação da patologia demonstrando a necessidade de identificar focos de potenciais epidemias para que os órgãos de saúde pública tomem atitudes compatíveis com suas realidades, a exemplo chama-se atenção Barbalha, Crato e Juazeiro do Norte seguidas pelo segundo grupo de Cidades Mauriti, Brejo Santo e Milagres que tendenciam características de clusters. Não existem indícios de casos 
importados recentes, ou seja, há o fator limitante de importação de casos novos na expansão da epidemia. Sugere-se, portanto, fomentar a pesquisa, usar de artifícios para eliminar o protozoário e aproveitar os fatores que REFERÊNCIAS

1. TORTORA, G.J; FUNKE, B.R; CASE, C.L. Microbiologia. 10 edição. Artmed. 2012. 23-665.

2. WHO. Burden of disease. Disponível em: <http://www.who.int/leishmaniasis/burden/en/>. Acesso em:02 de dezembro de 2013a.

3. WHO. Burden of disease. Disponível em: <http://www.who.int/leishmaniasis/epidemic/en/> Acesso em:02 de dezembro de 2013b.

4. WHO. Geographical distribution of visceral leishmaniasis in the Old and New world. Geographical distribution of cutaneous and mucocutaneous leishmaniasis in the New World. Disponível em: <http://www.who.int/leishmaniasis/leishmaniasis_maps/en/i ndex.html > Acesso em:13 de fevereiro de 2014.

5. BRASIL; Ministério da Saúde; Secretaria de Vigilância em Saúde; Departamento de Vigilância Epidemiológica. Guia de vigilância epidemiológica. 7. ed. - Brasília MS. 2009.

6. BRASIL; Ministério da Saúde. Portaria $n^{\circ} 104$ de 25 de janeiro de 2011. 2011.

7. ARRUDA, MM. Leishmanioses. 2013. Disponível em: <http://www.zoonoses.org.br/absoluto/midia/imagens/zoono ses/arquivos_1258562831/6365_crmv-pr_manual-

zoonoses_leishmanioses.pdf> Acesso em: 10 de dezembro de 2013.

8. SOUZA, A.Q. Leishmaniose cutânea no ceará: aspectos históricos, clínicos e evolução terapêutica. [Tese de doutorado]. Fortaleza-CE. Curso de Doutorado em Farmacologia. Faculdade de Medicina. Universidade Federal do Ceará-UFC. 2009.

9. BARbosA, J.P.A. História da Saúde Pública do Ceará. Fortaleza. Edições UFC.1994.

10. BRASIL, M.M.A; IBAMA; PREVFOGO. Plano operativo de prevenção e combate aos incêndios florestais da floresta nacional de Araripe-Apodi. CratoCE. 2006.

11. FELIX, G.C; ARAÚJO NETO, F.B; BACURAU, F.R.S.; BRITO, L.A.; INÁCIO, N.T.D.; SOUSA, P.S. Perfil epidemiológico de pacientes com Leishmaniose tegumentar americana no município de Barbalha, CE. Id on Line Revista de Psicologia. Julho de 2011, v.1, n.14, p.30-35. ISSN 1981-1189.

12. CEARÁ. Governo do Estado. Disponível em: < http://www.ceara.gov.br/ >. Acesso em: 06 de janeiro de 2014. isolam a área para potencializar a erradicação da patologia, assim como se fez historicamente com a malária no Cariri Cearense.

13. INSTITUTO DE PESQUISA E ESTRATÉGIA ECONÔMICA DO CEARÁ -IPECE. Disponível em: <http://www2.ipece.ce.gov.br/atlas/capitulo2/21.htm >. Acesso em: 06 de janeiro de 2014.

14. SILVA, J.C.F. Leishmaniose Tegumentar Americana (LTA), nos municípios de Crato, Juazeiro do Norte e Barbalha, estado do Ceará, Brasil, 20032005.[Dissertação de Mestrado]. Curso de Mestrado em Patologia. Faculdade de Medicina. Universidade Federal do Ceará-UFC. Fortaleza-CE. 2008.

15. SCHRIEFER, A.L.F. et al. Papel do Parasita e do Hospedeiro na Expressão Clínica das Leishmanioses. Gazeta Médica da Bahia 2005;75(1):Jan-Jun:46-56.

16. DESJEUX $P$. The Increase in Risk Factors for Leishmaniasis worldwide. Transactions of the royal society of tropical medicine and hygiene. (95) 239-243. 2001.

17. MATOS, J. SESAU Juazeiro centraliza combate e controle a leishmaniose . 2011. Disponível em: <http://portal.conasems.org.br/index.php/comunicacao/muni cipio-em-foco/546-sesau-juazeiro-centraliza-combate-econtrole-a-leishmaniose >. Acesso: 12 de dezembro de 2013.

18. LIMA, M.B; BATISTA, E.A.R. Epidemiologia da leishmaniose visceral humana em Fortaleza-ce. 2010. RBPS 2009; 22 (1) : 16-23.

19. RALPH R FRERICHS. This site is devoted to the life and times of Dr. John Snow (1813-1858), a legendary figure in the history of public health, epidemiology and anesthesiology. UCLA. Department of Epidemiology. School of Public Health. Disponível em: <http://www.ph.ucla.edu/epi/snow.html\#ANESTHESIA >. Acesso em: 18 de fevereiro e 2014.

20. FREITAS, J.C.C; PINHEIRO, D.C.S.N. Aspectos celulares e moleculares da resposta imunitária a Leishmania spp. Faculdade de Veterinária, Programa de Pós-Graduação em Ciências Veterinárias da Universidade Estadual do Ceará, Fortaleza-Ceará. Revista portuguesa de ciências veterinárias. 2010, v.105, p.11-20.

21. FIOCRUZ. Leishmaniose. 2013. Disponível em: < http://www.agencia.fiocruz.br/leishmaniose >. Acesso em outubro de 2013. 